\title{
Clasificación Oxford para la validación de un protocolo de antibioticoterapia subcutánea paliativa
}

\author{
Miguel Antonio Sánchez-Cárdenas* \\ Laura Camila Pulido-Garzón ** \\ Leydy Viviana Santamaría-Orozco** \\ Lina Katerine Rodríguez-Laverde** \\ Mónica Andrea Preciado-Vargas ${ }^{* *}$ \\ Sergio Daniel Ruíz-Alarcón** \\ Yeins Stiven Prado-Ariza**
}

Introducción: los casos de pacientes con procesos infecciosos al final de la vida muestran la necesidad de contar con alternativas que garanticen el cuidado y el manejo terapéutico instaurado. Las vías clásicas intravenosa, intramuscular y oral se ven limitadas, mientras que la vía subcutánea demuestra ser una alternativa prometedora; sin embargo, la escasa evidencia científica reflejada en el bajo número de investigaciones, devela la necesidad de explorar y generar productos científicos que respalden esta práctica. Metodología: estudio documental, con base en un proceso de revisión sistemática, en el cual se realiza la búsqueda de 10 acciones descritas en un protocolo. Fueron seleccionados 34 artículos en idioma inglés y español, excluyendo 17. Los artículos fueron clasificados a partir de la escala del Centre for Evidence-Based Medicine, Oxford, consultando las bases: Nursing Skills, Clinicalkey, Pubmed, Springerlink, Science Direct, ProQuest y Cochrane. Se utilizaron como descriptores: antibacterianos, cuidados paliativos, protocolos, catéter, subcutáneo, signos, tiempo, antibiótico, enfermería, registros, consentimiento informado, valoración. Resultados: el $60 \%$ de las acciones propuestas en el protocolo no tuvo artículos que respaldaran su práctica, solamente el $40 \%$ fue clasificado. Conclusión: el protocolo no se recomienda, puesto que más del $50 \%$ de las actividades no cuentan con evidencia científica que las respalde.

PALABRAS CLAVE: antibacterianos, cuidados paliativos, protocolos, tejido subcutáneo.

Para citar este artículo / Para citar este artigo/ To reference this article /

Sánchez-Cárdenas MA, Pulido-Garzón LV, Santamaría-Orozco LV, Rodríguez-Laverde MA, Ruíz-Alarcón. Prado-Ariza YS. Clasificación Oxford para la validación de un protocolo de antibioticoterapia subcutánea paliativa. Rev. cienc. cuidad. 2017; 14(1): 95-110.

\footnotetext{
* Enfermero. Magister en Administración en Salud. Doctor en Bioética. Líder Grupo de Investigación Salud Sexual y Reproductiva Enfermería UN BOSQUE. Director de Investigación y Proyección Social. Universidad El Bosque. Bogotá D.C.; Colombia. Correo electrónico: sanchezcmiguel@ unbosque.edu.co

** Estudiantes de Enfermería. Universidad El Bosque. Bogotá D.C.; Colombia. Correo electrónico: lpulidog@ unbosque.edu. co,1santamariao@ unbosque.edu. co,1krodriguezl@ unbosque.edu. co,mpreciado@ unbosque.edu.co, sruiza@unbosque. edu.co,yprado@ unbosque.edu.co *** Descriptores en Ciencias de la Salud (DeCS), en la página http://decs. bvs.br/E/homepagee. $\underline{\mathrm{htm}}$ de la Biblioteca Virtual en Salud del proyecto BIREME, de la Organización Mundial de la Salud y de la Organización Panamericana de Salud.
} 


\section{Oxford classification for the validation of a protocol of palliative subcutaneous antibiotic therapy}

\section{ABSTRACT}

Introduction: the cases of patients with infectious processes at the end of life show the necessity to count with alternatives that guarantee the care and the established therapeutic management. The classic intravenous, intramuscular and oral routes are limited, while the subcutaneous route demonstrates to be a promising alternative; however, the scarce scientific evidence reflected in the low number of research, unveils the necessity to explore and generate scientific products to endorse this practice. Methodology: a documental study, based on a systematic review process, in which the search of 10 actions described in a protocol is performed. 34 articles in English and Spanish were selected, excluding 17. The articles were classified based on the scale from Centre for Evidence-Based Medicine, Oxford, consulting the databases: Nursing Skills, Clinicalkey, Pubmed, Springerlink, ScienceDirect, ProQuest and Cochrane. The used descriptors: antibacterials, palliative care, protocols, subcutaneous catheter, signs, time, antibiotic, nursing, records, informed consent, and valuation. Results: $60 \%$ of the actions proposed in the protocol did not have articles to endorse their practice, only $40 \%$ was classified. Conclusion: the protocol is not recommended, since more than $50 \%$ of the activities do not have scientific evidence to endorse them.

KEYWORDS: anti-bacterial agents, palliative care, protocols, subcutaneous tissue. 


\section{Classificação Oxford para a validação de um protocolo de aplicação paliativa de antibióticos por via subcutânea}

\section{RESUMO}

Introdução: os casos de pacientes com processos infecciosos ao final da vida mostram a necessidade de contar com alternativas que garantam o cuidado e o manejo terapêutico estabelecido. As vias clássicas intravenosa, intramuscular e oral estão limitadas, enquanto que a via subcutânea demostra ser uma alternativa prometedora; porém, a escassa evidencia científica refletida no baixo número de pesquisas, revela a necessidade de explorar e gerar produtos científicos que respaldem esta prática. Metodologia: estudo documental, baseado num processo de revisão sistemática, no qual se realizou a busca de 10 ações descritas num protocolo. Foram selecionados 34 artigos em idioma inglês e espanhol, excluindo 17. Os artigos foram classificados a partir da escala do Centre for Evidence-Based Medicine, Oxford, consultando as bases: Nursing Skills, Clinicalkey, Pubmed, Springerlink, Science Direct, ProQuest e Cochrane. Utilizaram-se como descritores: antibacterianos, cuidados paliativos, protocolos, cateter, subcutâneo, signos, tempo, antibiótico, enfermagem, registros, consentimento informado, valoração. Resultados: o $60 \%$ das ações propostas no protocolo não teve artigos que respaldaram sua prática, somente o $40 \%$ foi classificado. Conclusão: o protocolo não se recomenda, já que mais do $50 \%$ das atividades não têm evidencia científica para apoiá-lo.

PALAVRAS-CHAVE: antibacterianos, cuidados paliativos, protocolos, tela subcutânea. 


\section{INTRODUCCIÓN}

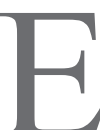
n la actualidad, el desarrollo de la práctica clínica muestra de manera constante una alta variabilidad existente e injustificada en la prestación de servicios de salud, por lo que la evidencia de la práctica clínica evidencia, de forma amplia, cómo desde diferentes instituciones se aborda de manera diferente un mismo evento patológico o terapéutico (1). Esto hace que se creen protocolos como herramientas que facilitan la toma de decisiones, que describen el cuidado apropiado basado en la evidencia científica y en dirección a la mejora de la calidad asistencial, para llegar a acuerdos en temas de conflictos y discrepancias, reduciendo así la variabilidad de los cuidados (2).

El ejercicio profesional de los actores del sistema de salud y el mismo propio de enfermería ha estado inmerso en un fenómeno de amplia magnitud descrito hoy en día como variabilidad clínica. Este fenómeno es posible de percibir en cada paso del proceso de atención, siendo el sujeto aceptor de los servicios de salud el más afectado. Esto favorece una atención pobre en estándares de calidad, con déficits en la atención segura del paciente (3).

La práctica clínica heterogénea favorece que los distintos profesionales y personal vinculado a los servicios de salud desarrolle actividades bajo un estilo libre, fuera de bases fundamentadas en evidencia científica que respalden cada parte del proceso. Una de las causas de este hecho relaciona la incertidumbre como causa primaria de este fenómeno, generado por evidencia científica ambigua e incompleta, que no permite un desarrollo efectivo y de calidad de la práctica (4-5).

Los patrones de buenas prácticas clínicas y los estudios frente a este fenómeno deben orientarse a la mejora de la seguridad del paciente, reduciendo la incertidumbre científica y mejorando la ética, con el fin de brindar la mejor atención en salud disponible. En la actualidad, las guías de práctica y los protocolos clínicos surgen como una alternativa altamente efectiva, que garantiza normalizar la práctica en aras de controlar este fenómeno, que se supone existe, pero que en muchos casos se desconoce (3-4).

De acuerdo al Global Atlas of Palliative Care at the End of Life (6), los cuidados paliativos se han descrito para la intervención de individuos con un amplio grupo de enfermedades crónicas y/o degenerativas, entre las que se encuentran: alzhéimer y otras demencias, cáncer, enfermedades cardiovasculares, trastornos hepáticos como cirrosis, diabetes, VIH/SIDA, falla renal, esclerosis múltiple, enfermedad parkinsoniana, artritis reumatoide y tuberculosis.

Amrallah et al. (7) describen que una de las principales comorbilidades presentadas en individuos requirentes de cuidados paliativos es el desarrollo de estados infecciosos, secundario al proceso fisiopatológico propio de la enfermedad y a los procedimientos terapéuticos realizados, muchos de ellos invasivos, lo que predispone y facilita la colonización de agentes microbianos causantes de enfermedades infecciosas, capaces de agudizar el estado clínico del individuo. Estos autores sostienen que pacientes con enfermedad oncológica en fase terminal, progresiva e incurable, suelen ser más susceptibles y vulnerables al desarrollo de procesos infecciosos de alta variabilidad etiológica.

Estudios clínicos (7) han demostrado una alta prevalencia de colonización por agentes oportunistas Gram-negativos en enfermedades infecciosas padecidas por pacientes en estados terminales, siendo los principales sitios de infección: vías respiratorias con compromiso de grandes regiones del árbol bronquial, sistema urinario, infecciones del sitio quirúrgico debido, en su mayoría, a un pobre proceso de cicatrización y en mayor incidencia piel y tejidos subcutáneos. Estas infecciones son la causa más frecuente de comorbilidad, sumada a la inmovilidad, el deterioro cognitivo y el uso de catéteres y sondas, lo que predispone a que este tipo de pacientes presenten una mayor susceptibilidad y un mayor riesgo al desarrollo de procesos infecciosos (7).

Según Soler et al. (8), la intervención terapéutica debe apuntar al inicio temprano de tratamientos sintomatológicos y antimicrobianos que mejoren la calidad de vida del paciente y eviten el desarrollo de estados clínicos agudos (8). Para Gallardo y Gamboa (9), la ceftriaxona, como una cefalosporina de tercera generación, es uno de los antibióticos que ha presentado mayor uso clínico en la administración por vía subcutánea -evaluado en ensayos clínicos-, que se sugiere, por datos de eficacia y tolerabilidad, para su uso en pacientes con imposibilidad de utilizar la vía oral y endovenosa, seguidos de cefepime, ampicilina y teicoplanina (9).

Sin embargo, la baja evidencia científica y la poca profundidad de los estudios clínicos observada en 
investigaciones orientadas a la búsqueda de nuevas alternativas dirigidas a pacientes en fases terminales, genera la necesidad de reformular el desarrollo de terapias novedosas que favorezcan el abordaje clínico $\mathrm{y}$, a su vez, garanticen una mejora en la calidad de vida de estos pacientes. Por tal motivo, Soler et al. (8) proponen evaluar la eficacia terapéutica y la seguridad del tratamiento antimicrobiano por vía subcutánea en pacientes con infecciones paralelas a estados patológicos terminales, además de enfatizar las investigaciones en la identificación de efectos adversos presentados posterior a la administración por esta vía y su actividad farmacocinética tras la administración de compuestos antimicrobianos.

Hernández y García (10) mencionan que la vía de elección que predomina en el tratamiento sintomatológico de pacientes requirentes de cuidados paliativos es la vía oral (10); no obstante, se ha notado que en muchas ocasiones esta vía se imposibilita debido al estado clínico del paciente, siendo necesario recurrir a otras vías alternas.

Existen diferentes causas por las cuales se puede perder el uso de la vía oral. Hernández y García (10) describen las siguientes: intolerancia gástrica, imposibilidad deglutoria, náuseas y vómitos persistentes, intolerancia a opioides orales y la necesidad de administrar dosis más altas, mala absorción, debilidad extrema, estados confusionales, agonía, disminución del estado de consciencia y obstrucción intestinal, entre otros factores. En estos casos, vías alternas a la oral pueden ser de gran utilidad para el abordaje de estos pacientes, favoreciendo ampliamente la intervención temprana y oportuna, y más aún, promoviendo la garantía de la calidad de vida en estos individuos.

Gallardo y Gamboa (9) afirman que, en el caso de la vía intravenosa, los servicios de salud se enfrentan a la necesidad de personal entrenado para su empleo, la dificultad para instaurar un acceso venoso así como para su mantenimiento, por ejemplo, en pacientes caquéxicos -con una pérdida marcada de masa magra-, siendo eventualmente crítico para el proceso, pues la administración domiciliaria y las frecuentes complicaciones, como infecciones y limitaciones, suponen una problemática asistencial al generar una menor autonomía y mayor costo, además de no garantizar la calidad de los cuidados (9). La vía rectal puede resultar una buena opción ante los problemas de disfagia o de absorción; sin embargo, a pesar de su bajo costo, hay pocos fármacos que se puedan emplear por dicha vía debido a la gran variabilidad farmacocinética que se presenta (9).

En cuanto a la vía intramuscular se presenta una gran problemática, pues debido a la alta innervación muscular su acceso genera un alto pico doloroso, lo que puede agudizar el cuadro clínico mismo del paciente, sumado, además, a la pérdida muscular en pacientes caquéxicos, lo que imposibilita su uso (9). La vía transdérmica podría ser otra opción ante la imposibilidad o dificultad de empleo de la vía oral o intravenosa, pero presenta desventajas, al igual que la vía rectal, relacionadas con la actividad farmacocinética del medicamento administrado, entre las cuales se encuentra el retraso de hasta 12 a 24 horas para alcanzar un estado estacionario, lo que conlleva un difícil control del dolor dentro de las primeras 72 horas en pacientes de cuidados paliativos (9). Se agrega el hecho de presentar un período considerable de continuación del efecto tras su retirada y una gran variabilidad individual (11), razón por la cual esta vía de administración es preferible utilizarla para el control del dolor estable, pero con dosis bajas de opiáceos (9).

Según Gallardo y Gamboa (9), la vía subcutánea supone una alternativa en situaciones en las que no resulta apropiado el uso de la vía oral, intravenosa o intramuscular, en pacientes requirentes de cuidados paliativos. La vía subcutánea no presenta barreras de absorción, es decir, se evita el efecto de primer paso al entrar directamente en el torrente sanguíneo. Al administrar un medicamento por esta vía, su contenido se extiende por toda la superficie corporal y no supone una limitación del acceso, como puede ocurrir con la vía intravenosa o intramuscular (9).

Según Macmillan et al. (12), la vía subcutánea resulta tan efectiva como la vía intravenosa, pero con la ventaja de ser menos invasiva. Además de esto, la vía subcutánea plantea una opción segura, económica, sencilla y menos agresiva para el paciente, permitiendo mayor comodidad y con ello probablemente una mejor calidad de vida. A partir de este planteamiento se fundamenta el sentido de la presente investigación, que apunta a la búsqueda de fuentes de información e investigaciones que respalden el uso de esta vía. Sin embargo, se debe tener en cuenta que no todos los contenidos que se encuentran en los artículos
ISSN 1794-9831

E-ISSN 2322-7028

Vol. 14 No. 1

Ene - Jun 2017

Cúcuta, Colombia 
E-ISSN 2322-7028

Vol. 14 No. 1

Ene - Jun 2017

Cúcuta, Colombia científicos publicados tienen el mismo valor o impacto en cuanto a la calidad; por tal motivo, desde años atrás se ha venido clasificando este tipo de documentos, dándoles validez científica para ser adoptados o acogidos en instituciones para beneficio de los usuarios.

Por lo anterior, se deben conocer las herramientas, instrumentos y escalas que clasifican, jerarquizan y valoran la evidencia disponible de la hipodermoclisis, de forma tal que, con base en su utilización, se puedan emitir juicios de recomendación. Adicionalmente, se deben tener en cuenta los grados de recomendación que son aquellos que clasifican la sugerencia de adoptar o no la adquisición o puesta en marcha de tecnologías en salud, según el rigor científico de cada tipo de diseño (13). En la actualidad se dispone de tres tipos de clasificación: por niveles de evidencia, por grados de recomendación y mixtos.

Según Manterola y Zabando (13), una de las escalas más completas es la escala Centre for Evidence-Based Medicine, Oxford (OCEBM), en la que se tienen en cuenta no sólo las intervenciones terapéuticas y preventivas, sino también las ligadas al diagnóstico, el pronóstico, los factores de riesgo y la evaluación económica (14). Esta clasificación tiene la ventaja de asegurar el conocimiento más atingente a cada escenario, por su alto grado de especialización. Además, tiene el beneficio de aclarar cómo afecta la falta de rigurosidad metodológica el diseño de los estudios, disminuyendo su valoración no sólo en la gradación de la evidencia, sino también en la fuerza de las recomendaciones (13).

Esta escala se caracteriza por valorar la evidencia según el área temática o escenario clínico y el tipo de estudio que involucra al problema clínico en cuestión. Su ventaja radica en medir la evidencia de acuerdo con el mejor diseño para cada escenario clínico, otorgándole intencionalidad y agregando la revisión sistemática ${ }^{\dagger}(\mathrm{RS})$ en los distintos ámbitos. Así, por ejemplo, al tratarse de un escenario clínico relacionado con pronóstico de un evento de interés, la evidencia será valorada a partir de una RS de estudios de cohortes con homogeneidad o, en su defecto, de estudios de cohortes individuales con un seguimiento

$\uparrow$ Diseño de investigación observacional y retrospectivo, que sintetiza los resultados de múltiples investigaciones primarias (13). superior al $80 \%$ de la cohorte. En cambio, si el escenario se refiere a terapia o tratamiento, la evidencia se valorará principalmente a partir de RS de ensayos clínicos (EC) o, en su defecto, de EC individuales con intervalos de confianza estrechos (13).

A pesar de que la hipodermoclisis (método de administración de fluidos y de medicación a nivel del tejido celular subcutáneo) es una técnica segura, sencilla, efectiva y relativamente cómoda para el paciente, según Hernández et al. (10), en Colombia siguen siendo aislados los estudios de investigación encaminados a la búsqueda de alternativas terapéuticas en pacientes requirentes de cuidados paliativos $\mathrm{y}$, más aún, en estudios dirigidos a la evaluación de la vía subcutánea; sin embargo, se muestra una baja evidencia científica en otros países latinoamericanos, europeos y orientales.

\section{METODOLOGÍA}

Se desarrolló un estudio documental en el año 2016, con base en un proceso de revisión sistemática, desarrollando cada fase de la siguiente manera:

\section{Fase I: Definición del problema}

En una IPS de Bogotá se identifica como problema la falta de evidencia científica y grado de recomendación de las acciones propuestas en un protocolo de antibióticoterapia subcutánea, lo que lleva a una investigación exhaustiva de artículos que incluyan criterios que aporten a ésta. Así mismo, se determinó que la unidad de análisis corresponde a los niveles de evidencia y grados de recomendación que tiene cada una de las acciones, las cuales componen la unidad de investigación.

\section{Fase II: Localización y selección de artículos}

Se realizó una identificación de artículos potenciales con la estandarización de criterios de inclusión, dentro de los cuales se estableció ampliar la ventana de tiempo a 15 años, correspondiente a los periodos comprendidos entre los años 2000 y 2015, dado que al realizar el estado de la cuestión se evidenció escasez de estudios en un periodo de 10 años. También se incluyeron artículos que no tuvieran alto factor de impacto pero que se consideraran con información importante para la investigación y que plantearan experiencias por parte de profesionales de enfermería. 
Adicionalmente, se aplicaron criterios de idioma de las publicaciones, tales como inglés y español ${ }^{\dagger \dagger}$.

Una vez identificados los criterios para la selección de artículos potenciales, se incluyeron en bases de datos de revistas científicas con mayor fuente de artículos de enfermería clínica, siendo las más reconocidas y respaldadas respectivamente: Nursing Skills, como una amplia combinación de competencias y contenidos de enfermería; Embase, como base de datos especializada en farmacología; Science Direct, especialista en textos de revistas científicas; Clinicalkey, especializada en revistas médicas y quirúrgicas con procedimientos indexados y Springerlink como libro electrónico con 12 áreas de conocimientos.

Así mismo, se establecieron las siguientes palabras claves: catéter, subcutáneo, signos, tiempo, antibiótico, enfermería, registros, consentimiento informado y valoración, normalizadas por medio de Descriptores en Ciencias de la Salud (DECS) que, sumados a las operaciones lógicas, se aplicaron para realizar la búsqueda individualizada. Puede haber varias estrategias de búsqueda debido a que éstas se hacen en función de la acción del protocolo.

Luego de la búsqueda se realizó un esquema de selección que incluyó los criterios a evaluar de cada artículo, tomando inicialmente el resumen y la metodología de cada uno para poder clasificarlos de acuerdo con la escala de jerarquización elegida. Lo anterior se realizó en parejas y de manera independiente para aumentar la fiabilidad. Al momento de incluir los criterios a evaluar para cada artículo, fueron seleccionados 17 , un número muy reducido y de poca información para la realización del proyecto de investigación.

\section{Fase III: Selección del instrumento de evaluación de la evidencia}

Al tener seleccionados los artículos, se utilizaron los datos de los estudios relevantes. Esto se hizo con la jerarquización establecida de la escala que fue elegida previamente por los miembros de la investigación. Se realizó una comparación esquemática de tres escalas de medición de niveles de evidencia y grados

† Nota: dada la importancia de tres artículos consultados, los cuales no se encuentran dentro de la ventana de tiempo seleccionada, se decidió incluirlos en la investigación. de recomendación que se relacionen con el escenario clínico de la investigación, según el escenario de tratamiento, siendo en este caso National Institute for Heatlh and Clinical Excellence (NICE), Sackett y OCEBM, identificando ventajas y desventajas. Una vez debatidas estas escalas, se estableció que, por ser transversal en todos los escenarios, ser más reciente y tener mayor aplicabilidad objetiva en la población diana, la escala que se tendría en cuenta para la evaluación objetiva de cada artículo sería la OCEBM.

\section{Fase IV: Análisis de la información}

Posteriormente, se llevó a cabo el plan de análisis de la información por medio del cual se realizó la revisión de los artículos científicos a través de evaluación binaria, un método en el que dos investigadores realizan la lectura de un estudio y discuten según la escala OCEBM a qué nivel de evidencia y grado de recomendación pertenece el artículo. Si la perspectiva de los dos miembros investigadores frente a la metodología no es similar, entraría un tercer investigador a dirimir la diferencia. Para lo anterior se utilizó un formato de fichas de captura establecidas por la facultad de enfermería de una institución de educación superior en Bogotá, en la cual se describen brevemente los datos bibliográficos de la investigación tales como autores, tipo de documento y de estudio, palabras clave y ubicación del estudio, entre otros, y se realiza un breve resumen que describe el objetivo, materiales y métodos, principales resultados y conclusiones, esto con el objetivo de recolectar los datos más relevantes del documento.

Se realizó la búsqueda bibliográfica en el periodo comprendido entre los meses de enero a mayo del año 2015, correspondiente a diez acciones descritas en el protocolo en consideración a la importancia para la ejecución del proceso de administración de antibióticos. En la tabla 1 se muestra la búsqueda y recolección de artículos por cada acción, teniendo en cuenta que por cada una se seleccionaron términos que fueron normalizados y, posteriormente a esto, se realizó la construcción de términos con los operadores booleanos y búsqueda en bases de datos.

En la etapa de recolección de artículos fueron seleccionados un total de 36, de los cuales 17 fueron excluidos del estudio puesto que no aplicaban dentro
ISSN 1794-9831

E-ISSN 2322-7028

Vol. 14 No. 1

Ene - Jun 2017

Cúcuta, Colombia 
E-ISSN 2322-7028

Vol. 14 No. 1

Ene - Jun 2017

Cúcuta, Colombia de los criterios de inclusión determinados por el grupo investigador durante el desarrollo metodológico, motivo por el cual fueron incluidos un total de 19 artículos para el desarrollo de la investigación.

En cuanto a limitaciones del presente estudio, se identificó que, al realizar la búsqueda bibliográfica, aún siguen siendo limitados los estudios de investigación con alta evidencia científica dirigidos al manejo de vías alternativas para el manejo de pacientes de cuidados paliativos como lo es la vía subcutánea. 
Tabla 1. Búsqueda y recolección de artículos.

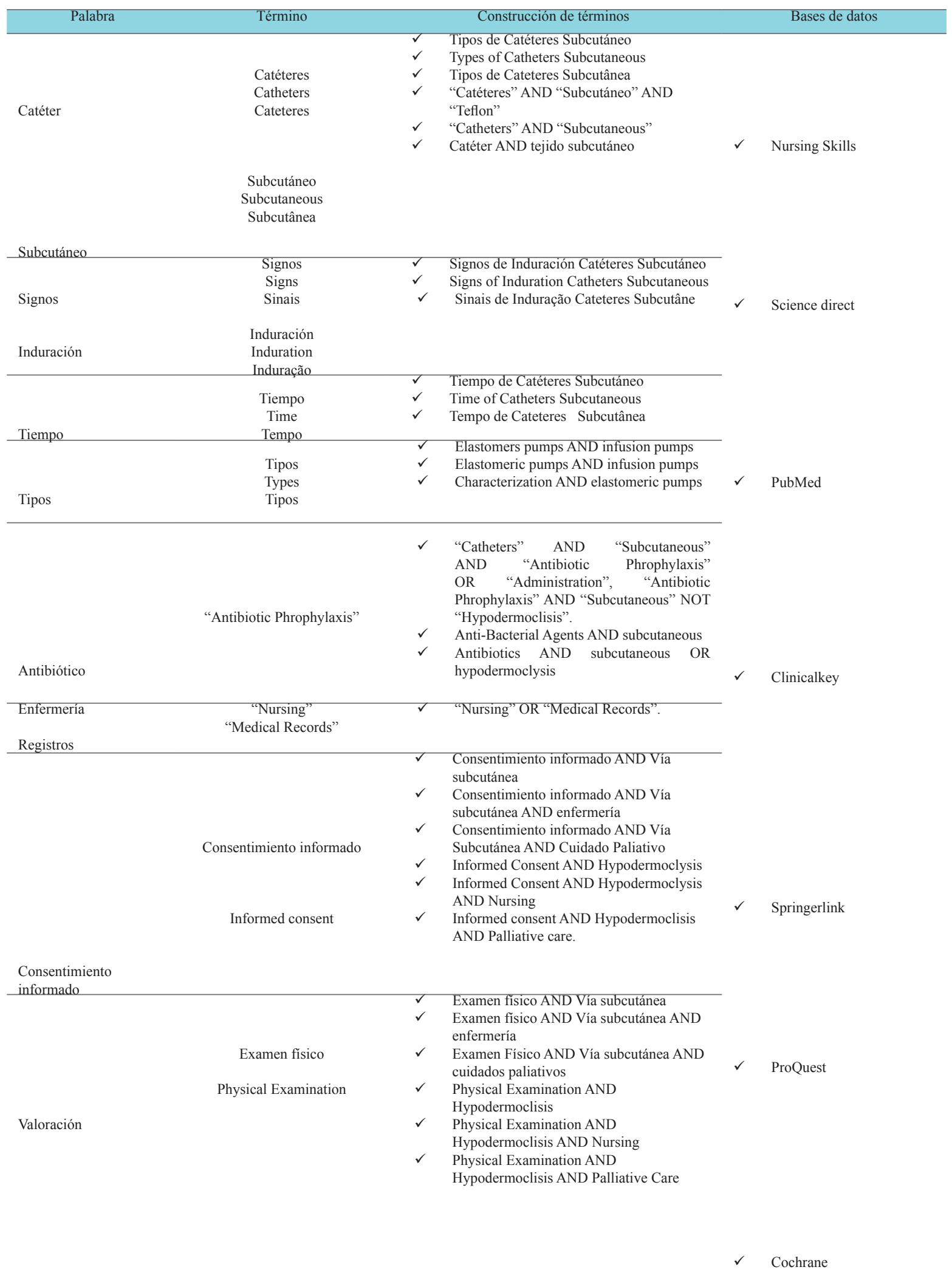

ISSN 1794-9831

E-ISSN 2322-7028

Vol. 14 No. 1

Ene - Jun 2017

Cúcuta, Colombia

Fuente: elaboración propia. 
E-ISSN 2322-7028 Vol. 14 No. 1 Ene - Jun 2017 Cúcuta, Colombia

\section{Niveles de evidencia y grados de recomendación}

Los niveles de evidencia y grados de recomendación de las diez acciones del protocolo de antibioticoterapia subcutánea que fueron seleccionadas según la escala de OCEBM se muestran en la tabla 2, donde se puede evidenciar que en el $60 \%$ de ellas no se encontró literatura que reportara evidencia científica, mientras que en el $40 \%$ restante se encontró un total de 17 artículos a los cuales se les realizó su respectiva clasificación. 
Tabla 2. Niveles de evidencia y grados de recomendación.

ISSN 1794-9831

E-ISSN 2322-7028

Vol. 14 No. 1

Ene - Jun 2017

\begin{tabular}{|c|c|c|c|c|}
\hline Actividad & Artículo & Autor (es) & $\begin{array}{l}\text { Nivel de } \\
\text { evidencia }\end{array}$ & $\begin{array}{l}\text { Grado de } \\
\text { recomenda- } \\
\text { ción }\end{array}$ \\
\hline $\begin{array}{l}\text { 1. Consentimiento } \\
\text { informado }\end{array}$ & No se encontró literatura que reporte evidencia sobre esta actividad & & NA & NA \\
\hline $\begin{array}{l}\text { 2. Valoración de } \\
\text { enfermería para } \\
\text { instalación de catéter } \\
\text { subcutáneo }\end{array}$ & No se encontró literatura que reporte evidencia sobre esta actividad & & NA & NA \\
\hline $\begin{array}{l}\text { 3. Técnica aséptica } \\
\text { antes de realizar } \\
\text { procedimiento }\end{array}$ & No se encontró literatura que reporte evidencia sobre esta actividad & & NA & NA \\
\hline $\begin{array}{l}\text { 4. Técnica de } \\
\text { instalación del } \\
\text { catéter subcutáneo }\end{array}$ & No se encontró literatura que reporte evidencia sobre esta actividad & & NA & NA \\
\hline \multirow[t]{2}{*}{ 5. Tipos de catéter } & $\begin{array}{l}\text { A prospcetive comparison study between a butterfly needle and tef- } \\
\text { lon canula for subcutaneous narcotic administration. }\end{array}$ & Macmillan et al. (12) & $2 b$ & B \\
\hline & $\begin{array}{l}\text { A prospective, within- patient comparison between metal butterfly } \\
\text { needles and teflon cannulae in subcutaneous infusion of drugs to } \\
\text { terminally ill hospice patients. }\end{array}$ & Ross et al. (15) & $2 b$ & B \\
\hline \multirow{4}{*}{$\begin{array}{l}\text { 6.Uso de } \\
\text { dispositivos al } \\
\text { momento de la } \\
\text { inserción (Bombas } \\
\text { elastoméricas) }\end{array}$} & Reservorio venoso subcutáneo. & Álvarez et al. (16). & $2 b$ & B \\
\hline & $\begin{array}{l}\text { Stability of meropenem in polyvinly chloride bags and an } \\
\text { elastomeric infusion device. }\end{array}$ & Smith et al. (17). & $2 \mathrm{c}$ & B \\
\hline & $\begin{array}{l}\text { Protocolo de uso de reservorio subcutáneo en una unidad de } \\
\text { ginecología oncológica. }\end{array}$ & González et al. (18) & 5 & $\mathrm{D}$ \\
\hline & Transition to an elastomeric infusion pump in home care. & Broadhurst (19) & 5 & $\mathrm{D}$ \\
\hline $\begin{array}{l}\text { 7. Tiempo de } \\
\text { instalación de un } \\
\text { catéter }\end{array}$ & No se encontró literatura que reporte evidencia sobre esta actividad & & NA & NA \\
\hline $\begin{array}{l}\text { 8. Registros de } \\
\text { enfermería }\end{array}$ & $\begin{array}{l}\text { La trascendencia que tienen los registros } \\
\text { enfermería en el expediente clínico. }\end{array}$ & Méndez et al. (20) & 5 & $\mathrm{D}$ \\
\hline $\begin{array}{l}\text { 9. Valoración catéter } \\
\text { posterior inserción }\end{array}$ & No se encontró literatura que reporte evidencia sobre esta actividad & & NA & NA \\
\hline \multirow{10}{*}{$\begin{array}{l}\text { 10. Tipo de } \\
\text { medicamentos que } \\
\text { se administran por } \\
\text { catéter subcutáneo } \\
\text { (Antibióticos) }\end{array}$} & Subcutaneous Administration of Cefepime. & Walker et al. (21) & $2 b$ & B \\
\hline & 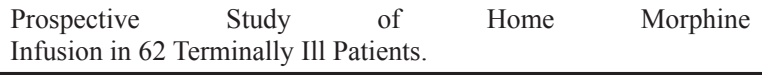 & Smeets et al. (22) & $2 b$ & B \\
\hline & 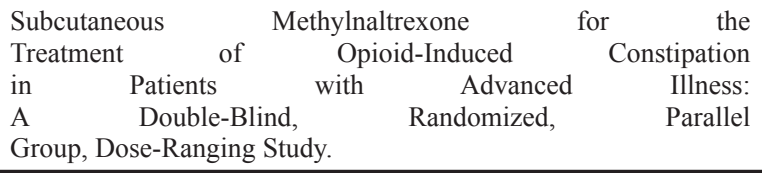 & Portenoy et al. (23) & $1 \mathrm{~b}$ & A \\
\hline & $\begin{array}{l}\text { The INFUSE-Morphine IIB Study: Use of Recombinant } \\
\text { Human Hyaluronidase (rHuPH20) to Enhance the } \\
\text { Absorption of Subcutaneous Morphine in Healthy } \\
\text { Volunteers. }\end{array}$ & Thomas et al. (24) & $1 \mathrm{~b}$ & A \\
\hline & Hipodermoclisis en pacientes con cáncer terminal. & Hernández et al. (25) & 4 & $\mathrm{C}$ \\
\hline & $\begin{array}{l}\text { Pharmacokinetics of ertapenem following intravenous and } \\
\text { subcutaneous infusion in patients. }\end{array}$ & Frasca et al. (26) & $1 \mathrm{~b}$ & A \\
\hline & $\begin{array}{l}\text { Subcutaneous administration of rituximab (MabThera) and } \\
\text { trastuzumab (Herceptin) using hyaluronidase. }\end{array}$ & $\begin{array}{l}\text { Shpilberg y Jackisch } \\
(27)\end{array}$ & $2 b$ & B \\
\hline & $\begin{array}{l}\text { Administración de medicamentos por vía subcutánea en cuidados } \\
\text { paliativos. }\end{array}$ & Matoses et al. (28) & 5 & $\mathrm{D}$ \\
\hline & $\begin{array}{l}\text { Subcutaneous and intravenous ceftriaxone administration in } \\
\text { patients more than } 75 \text { years of age. }\end{array}$ & Gauthier et al. (29) & $1 \mathrm{~b}$ & A \\
\hline & $\begin{array}{l}\text { Subcutaneous administration of drugs in palliative care: results of a } \\
\text { systemic observational study. }\end{array}$ & Bartz et al. (30) & $2 \mathrm{c}$ & B \\
\hline
\end{tabular}

Fuente: elaboración propia. 
E-ISSN 2322-7028

Vol. 14 No. 1

Ene - Jun 2017

Cúcuta, Colombia
En relación con las actividades que evidenciaron aporte científico se encuentran:

\section{Actividad 5. Tipos de catéter}

Se clasifican dos artículos $(12,15)$ con nivel de evidencia $2 \mathrm{~b}$ y grado de recomendación B. Durante la revisión de los respectivos artículos sobre la actividad, se identifica que uno de ellos (12) habla de la administración por vía subcutánea en cuidados paliativos de medicamentos del grupo de analgésicos, teniendo como metodología la comparación de dos agujas para la administración de estos medicamentos y siendo la cánula de teflón más cómoda para los pacientes pero a la vez incómoda para el personal de salud debido a la dificultad para la inserción, mientras que el otro plantea dos tipos de dispositivos para la infusión por vía subcutánea de medicamentos, pero en realidad ninguno de los dos habla de administración de antibioticoterapia; por tal motivo no aplican para el trabajo.

Actividad 6. Uso de dispositivos al momento de la inserción (bombas elastoméricas)

Se clasifican dos artículos (18-19) con nivel de evidencia 5 y grado de recomendación $\mathrm{D}$, siendo la clasificación más baja en la escala OXFORD y, por tal motivo, no aportan nivel científico a la investigación. Por otro lado, se encontraron dos artículo (16-17) con nivel de evidencia $2 \mathrm{~b}$ y grado de recomendación $\mathrm{B}$, es decir, estudio de cohortes o ensayo clínico aleatorizado de baja calidad y recomendación favorable. Teniendo en cuenta que los dos hablan sobre la administración de antibióticos por vía subcutánea, tratando uno de ellos del betalactamico meropenem, resultan significativos y con nivel científico pertinente para la investigación.

\section{Actividad 8. Registros de enfermería}

Se clasifica un artículo (20) con nivel de evidencia 5: opinión de expertos sin valoración crítica explicita, o basados en la fisiología, y grado de recomendación D: ni se recomienda ni se desaprueba, siendo este un estudio prospectivo observacional donde, a través de un periodo especifico, se llevó a cabo un seguimiento y verificaron de los registros de un grupo de enfermeras, evidenciando un desconocimiento en el $9.7 \%$ del grupo total de 62 enfermeras sobre lo relacionado con la importancia y el cómo diligenciar estos documentos. Sin embargo, el artículo no menciona registros relacionados con cuidados paliativos ni antibioticoterapia subcutánea $\mathrm{y}$, por tal motivo, no se considera de relevancia para esta investigación.

\section{Actividad 10. Tipo de medicamentos que se administran por catéter subcutáneo (antibióticos)}

Se encontraron 10 artículos (21-30), de los cuales $3(21,28,29)$ hablan acerca de administración de antibioticoterapia por vía subcutánea en cuidados paliativos. Uno de ellos (29) con nivel de evidencia $1 b$ y grado de recomendación A, es decir, ensayo clínico aleatorizado con intervalo de confianza estrecho y extremadamente recomendable: otro (21) con nivel de evidencia $2 \mathrm{~b}$ : estudio de cohortes o ensayo clínico aleatorizado de baja calidad y grado de recomendación B -recomendación favorable-, y otro (28) con nivel de evidencia 5: opinión de expertos sin valoración crítica explicita, o basados en la fisiología y grado de recomendación D: ni se recomienda ni se desaprueba, siendo los dos primeros artículos ensayos clínicos con alta evidencia científica para el trabajo investigativo.

En relación con la metodología que se llevó a cabo en el artículo de Walker et al. (21), se evidencia que el grupo poblacional a estudio está integrado por voluntarios sanos con un rango de edad de 1865 años y criterios de inclusión y exclusión que se requerían para poder ser viables para la investigación. Se administra $1 \mathrm{~g}$ de cefepime en $50 \mathrm{ml}$ de suero glucosado al $5 \%$ durante 30 minutos por bomba de infusión en una aguja mariposa de calibre 23 insertada por vía subcutánea a nivel abdominal. La concentración plasmática del cefepime fue analizada a partir del programa Non-Parametric Adaptive Grid (NPAG). Así, se puede identificar que la concentración plasmática a nivel subcutáneo es similar a la adquirida por vía intramuscular.

El segundo artículo de Gauthier et al. (29), menciona que el estudio se realizó a partir del análisis de bases de datos del software de gestión farmacéutica Pharma de los pacientes mayores de 75 años que recibieron administración de ceftriaxona durante la estancia hospitalaria a partir del 1 de enero al 31 de diciembre de 2011 en un hospital en Francia, teniendo en cuenta la administración de ceftriaxona con un intervalo de 24 horas por vía subcutánea o intravenosa. 
Por tanto, se infiere que los niveles plasmáticos de ceftriaxona por vía subcutánea son similares a los administrados por vía intravenosa. El estudio argumenta que hay pocos datos en relación con la administración de antibioticoterapia por vía subcutánea, resaltando que el enfoque de vía subcutánea se da más en población geriátrica (29).

La literatura reporta baja evidencia en la administración de antibióticos por vía subcutánea, en seis de las diez actividades que se analizaron del protocolo, referentes a: consentimiento informado, valoración de enfermería para instalación de catéter subcutáneo, técnica aséptica antes de realizar procedimiento, técnica de instalación del catéter subcutáneo, tiempo de instalación de un catéter y valoración del catéter posterior a la inserción.

En la práctica clínica, la vía subcutánea en los cuidados paliativos ha tenido mayor auge debido a las ventajas que garantiza en relación con el bienestar del paciente y su manejo por parte del personal de salud, pero que, a pesar de la existencia de protocolos, sigue existiendo variabilidad clínica en cuanto al manejo que requiere dicha vía. Se observa que sólo cuatro artículos: Frasca et al. (26), Matoses et al. (28), Gauthier et al. (29) y Bartz et al. (30) plantean, en el método, la administración de antibioticoterapia por vía subcutánea en cuidados paliativos, siendo sólo dos ensayos clínicos con un nivel de evidencia $1 \mathrm{~b}$ y grado de recomendación A, el nivel más alto de jerarquización de evidencia científica.

Es importante que los profesionales de enfermería empiecen a investigar sobre la antibioticoterapia subcutánea en pacientes requirentes de cuidado paliativo, con el fin de obtener mayor soporte teóricopráctico para las actividades que se estén realizando de manera empírica. Así, la variabilidad clínica disminuye y los cuidados brindados al paciente serán certeros.

\section{CONCLUSIONES}

Las actividades con mayor nivel de evidencia científica fueron: tipo de medicamentos que se administran por catéter subcutáneo. De diez artículos solamente dos hacen referencia a la antibioticoterapia con un grado de recomendación A y B. La segunda actividad con mayor cantidad de evidencia fue el uso de dispositivos al momento de la inserción, siendo útiles para la investigación solamente tres, con un grado de recomendación $\mathrm{B}$ y $\mathrm{D}$ según la escala de OCEBM, al igual que la actividad tipo de catéter, donde se encontraron dos artículos con el mismo grado de recomendación.

La actividad con menor cantidad de artículos y nivel de evidencia más bajo fue: registros de enfermería, siendo una revisión de la literatura, por lo cual se clasificó como 5D en la escala de OCEBM.

La antibioticoterapia subcutánea poco descrita en la literatura científica es relacionada, con mayor frecuencia, en población requirente de cuidados paliativos en casos en donde las vías de administración tradicionales muestran limitada disponibilidad secundaria a comorbilidades existentes y la situación clínica asociada.

La revisión del protocolo de administración antibioticoterapéutica subcutánea deja ver acciones y/o prácticas concernientes al procedimiento que, bajo una percepción de buena práctica clínica, muestran ser benéficas: Por tanto, éstas no ameritan la búsqueda activa de evidencia científica pues su aplicación ha sido demostrada como favorable para el paciente y han sido descritas en múltiples protocolos de práctica clínica.

\section{CONFLICTO DE INTERESES}

Los autores declaran no tener ningún conflicto de intereses.
ISSN 1794-9831

E-ISSN 2322-7028

Vol. 14 No. 1

Ene - Jun 2017

Cúcuta, Colombia 
Clasificación Oxford para la validación de un protocolo de antibioticoterapia subcutánea paliativa • Miguel Antonio Sánchez-Cárdenas, Laura Camila Pulido-Garzón, Leydy Viviana Santamaría-Orozco, Lina Katerine Rodríguez-Laverde, Mónica Andrea Preciado-Vargas, Sergio Daniel Ruíz-Alarcón, Yeins Stiven Prado-Ariza

ISSN 1794-9831

E-ISSN 2322-7028 Vol. 14 No. 1 Ene - Jun 2017 Cúcuta, Colombia

\section{REFERENCIAS BIBLIOGRÁFICAS}

1. Chinchilla MI, Salazar M, Calleja MA. Dispensación de medicación protocolizada en un servicio de urgencias de un hospital de tercer nivel. Farm Hosp. 2011 Junio; 35(3): 106-113.

2. Ochoa C. Diseño y evaluación de protocolos clínicos. Nuevo Hospital - Hospital Virgen de la Concha de Zamora. 2002; II(5): 1-21.

3. Fernández J, Richart M. Variabilidad de la práctica clínica en enfermería, una revisión integradora. Acta Paul Enferm [Internet]. 2012 [consultado 17 de julio de 2014]; 25(5): 809-816. Disponible en: http://www.scielo.br/ pdf/ape/v25n5/25.pdf

4. Wennberg JE. Unwarranted variations in healthcare delivery: implications for academic medical centres. BMJ [Internet]. 2002 [consultado 25 de mayo de 2014]; 325(7370): 961-964. Disponible en: http://www.ncbi.nlm. nih.gov/pmc/articles/PMC1124450/

5. Wennberg DE, Wennberg JE. Perspective: Addressing variations: is there hope for the future?. Health Affairs [Internet]. 2003 dic [consultado 22 de Abril de 2014]; p. 614-617. Disponible en: http://content.healthaffairs.org/ content/early/2003/12/10/hlthaff.w3.614.long

6. Worldwide Palliative Care Alliance (WPCA), World Health Organization (WHO). Global Atlas of Palliative Care at the End of Life. [Internet]. London: WPCA; 2014 [consultado 10 de febrero de 2015]. Disponible en: http://www.who.int/nmh/Global_Atlas_of_Palliative_Care.pdf

7. Amrallah M, Abdullah A, Mohamed S, Ahmad A, Ayman E, Amr E. The pattern of infection and antibiotics use in terminal cancer patients. J Egypt Natl Canc Inst [Internet]. 2014 [consultado 26 de mayo de 2014]; 26(3): 147-152. Disponible en: article/pii/S1110036214000405

http://www.sciencedirect.com/science/

8. Soler A, Santaeugenia S, Montané E. Antibióticos por vía subcutánea en pacientes que precisan cuidados paliativos. Med Clin (Barc). [Internet]. 2007 [consultado 25 de febrero de 2015]; 129(6): 236-7. Disponible en: http://www.ncbi.nlm.nih.gov/pubmed/17678607

9. Gallardo R, Gamboa F. Uso de la vía subcutánea en cuidados paliativos. [Internet]. España: Sociedad Española de Cuidados Paliativos (SECPAL); 2013 [consultado 22 de marzo de 2015]; 4(1 supl 2): Disponible en: http:// www.secpal.com $/ \% 5 \mathrm{CDocumentos} \% 5 \mathrm{CB} \log \% 5 \mathrm{Cmonografia}$ secpal_04.pdf

10. Hernández B, López C, García MA. Vía subcutánea. Utilidad en el control de síntomas del paciente terminal. MEDIFAM [en línea]. 2002 [consultado 22 de marzo de 2015]; 12(2): 104-110. Disponible en: http://scielo. isciii.es/pdf/medif/v12n2/colabora.pdf

11. Paix A, Coleman A, Less J, Grigson J, Brooksbank M, Thorne D, Ashby M. Subcutaneous fentanyl and sufentanil infusion substitution for morphine intolerance in cancer pain management. Pain [Internet]. 1995 [consultado 22 de mayo de 2015]; 63(2):263-9. Disponible en: http://www.ncbi.nlm.nih.gov/pubmed/8628593

12. Macmillan K, Bruera E, Kuehn N, Selmser P, Macmillan A. A prospective comparison study between a butterfly needle and teflon canula for subcutaneous narcotic administration. J Pain Symptom Manage [Internet]. 1994 [consultado 3 de Febrero de 2015]; 9(2): 82-84. Disponible en: http://www.sciencedirect.com/science/article/ pii/0885392494901600

13. Manterola DC, Zavando MD. Como interpretar los "niveles de evidencia" en los diferentes escenarios clínicos. Rev Chil Cir 2009; 61(6): 582-595.

14. Primo J. Niveles de evidencia y grados de recomendación (I/II). Enfermedad Inflamatoria Intestinal al día 2003; 2(2): 39-42.

15. Ross JR, Saunders Y, Cochrane M, Zeppetella G. A prospective, within-patient comparison between metal butterfly needles and Teflon cannulae in subcutaneous infusion of drugs to terminally ill hospice patients. Palliat Med [Internet]. 2002 [consultado 10 de febrero de 2015]; 16(1): 13-6. Disponible en: http://www.ncbi.nlm.nih. gov/pubmed/11963447

16. Álvarez JA, Villalba WO, Encina WP, Vega RB. Reservorio venoso subcutáneo. Rev. Cir. Parag [Internet]. 2012 [consultado 23 de abril de 2015]; 36(2): 14-16. Disponible en: http://scielo.iics.una.py/scielo.php?pid=S2307$04202012000200003 \&$ script $=$ sci arttext\&tlng $=$ es

17. Smith DL, Bauer SM, Nicolau DP. Stability of meropenem in polyvinyl chloride bags and an elastomeric infusion device. Am J Health Syst Pharm [Internet]. 2004 [consultado 15 de marzo de 2015]; 61(16): 1682-5. Disponible en: http://www.ncbi.nlm.nih.gov/pubmed/15540479

18. González E, Álvarez J, Siruela MR, Sánchez C, Nuñez MJ. Protocolo de uso del reservorio subcutáneo en una unidad de ginecología oncológica. Enfermería Clínica [Internet]. 2011 [consultado 20 de febrero de 2015]; 21(4): 223-226. Disponible en: http://www.sciencedirect.com/science/article/pii/S1130862111000970 
19. Broadhurst D. Transition to an elastomeric infusion pump in home care: an evidence-based approach. J Infus Nurs [Internet]. 2012 [consultado 10 de marzo de 2015]; 35(3): 143-151. Disponible en: http://www.ncbi.nlm. nih.gov/pubmed/22498484

20. Méndez G, Morales MA, Preciado M, Ramírez MR, Sosa V, Jiménez M. La trascendencia que tienen los registros de enfermería en el expediente clínico. Rev Mex Enf Cardiol [Internet]. 2001 [consultado 12 de marzo de 2015]; 9(1-4): 11-17. Disponible en: http://www.medigraphic.com/pdfs/enfe/en-2001/en011-4c.pdf

21. Walker P, Neuhauser MN, Tam VH, Willey JS, Palmer JL, Bruera E, et al. Subcutaneous administration of cefepime. J Pain Symptom Manage [Internet]. 2005 [consultado 2 de marzo de 2015]; 30(2): 170-4. Disponible en: http://www.ncbi.nlm.nih.gov/pubmed/16125032

22. Smeets PM, Beusmans GH, Weber WE. Prospective study of home morphine infusion in 62 terminally ill patients. J Pain Symptom Manage [Internet]. 1999 [consultado 20 de abril de 2015]; 18(6): 390-400. Disponible en: http://www.ncbi.nlm.nih.gov/pubmed/10641464

23. Portenoy RK, Thomas J, Moehl ML, Tran D, Galasso FL, Stambler N, et al. Subcutaneous methylnaltrexone for the treatment of opioid-induced constipation in patients with advanced illness: a double-blind, randomized, parallel group, dose-ranging study. J Pain Symptom Manage [Internet]. 2008 [consultado 2 de mayo de 2016]; 35(5): 458-68. Disponible en: http://www.ncbi.nlm.nih.gov/pubmed/18440447

24. Thomas JR, Yocum RC, Haller MF, Flament J. The INFUSE-Morphine IIB study: use of recombinant human hyaluronidase (rHuPH20) to enhance the absorption of subcutaneous morphine in healthy volunteers. J Pain Symptom Manage [Internet]. 2009 [consultado 26 de febrero de 2015]; 38(5): 673-82. Disponible en: http:// www.ncbi.nlm.nih.gov/pubmed/19819667

25. Hernández A, Hall $\mathrm{C}$, Hernández A. Hipodermoclisis en pacientes con cáncer terminal. Rev cubana med [Internet]. 2011 [consultado 16 de febrero de 2015]; 50(2): 150-156. Disponible en: http://www.bvs.sld.cu/ revistas $/ \mathrm{med} / \mathrm{vol} 502211 / \mathrm{med} 05211 . \mathrm{htm}$

26. Frasca D, Marchand S, Petitpas F, Dahyot C, Couet W, Mimoz O. Pharmacokinetics of Ertapenem following Intravenous and Subcutaneous Infusions in Patients. Antimicrob Agents Chemothe [Internet]. 2010 [consultado 14 de marzo de 2015]; 54(2): 924-926. Disponible en: http://aac.asm.org/content/54/2/924.full.pdf

27. Shpilberg O, Jackisch C. Subcutaneous administration of rituximab (MabThera) and trastuzumab (Herceptin) using hyaluronidase. Br J Cancer [Internet]. 2013 [consultado 12 de febrero de 2016]; 109(6): 1556-61. Disponible en: http://www.ncbi.nlm.nih.gov/pubmed/24002601

28. Matoses C, Rodríguez FJ, Sanz G, Murcia AC, Morante M, Navarro A. Administración de medicamentos por vía subcutánea en cuidados paliativos. Farm Hosp [Internet]. 2015 [consultado 8 de marzo de 2015]; 39(2): 71-79. Disponible en: http://www.sefh.es/fh/146_fhpdf002.pdf

29. Gauthier D, Schambach S, Crouzet J, Sirvain S, Fraisse T. Subcutaneous and intravenous ceftriaxone administration in patients more than 75 years of age. Med Mal Infect [Internet]. 2014 [consultado 8 de marzo de 2015]; 44(6): 275-280. Disponible en: http://www.ncbi.nlm.nih.gov/pubmed/24932703

30. Bartz L, Klein C, Seifert A, Herget I, Ostgathe C, Stiel S. Subcutaneous administration of drugs in palliative care: results of a systematic observational study. J Pain Symptom Manage. [Internet]. 2014 [consultado 8 de marzo de 2016]; 48(4): 540-547. Disponible en: http://www.ncbi.nlm.nih.gov/pubmed/24766744

\section{BIBLIOGRAFÍA}

Alcolea MT, Oter C, Martín A. Enfermería Basada en la Evidencia. Orígenes y fundamentos para una práctica enfermera basada en la evidencia. Nure investigación [Internet]. 2011 [consultado 19 de abril de 2015]; (Suppl 52): 1-7. Disponible en: www.nureinvestigacion.es/OJS/index.php/nure/article/download/538/527

Aristil P. Principios básicos de la farmacología. McGraw Hill [libro en internet]. 2010 [consultado 13 de marzo de 2015]. Disponible en: http://booksmedicos.org/manual-de-farmacologia-basica-y-clinica/

Beltrán OA. Factor de impacto. Rev Col Gastroenterol [Internet]. 2006 [consultado 25 de mayo de 2015]; 21(01): 57-61. Disponible en: http://www.scielo.org.co/scielo.php?script=sci_arttext\&pid=S0120-99572006000100009

Beltrán OA. Revisiones sistemáticas de la literatura. Rev Col Gastroenterol [Internet]. 2005 [consultado 19 de abril de 2015]; 20(1): 60-69. Disponible en: http://www.scielo.org.co/pdf/rcg/v20n1/v20n1a09.pdf 
Clasificación Oxford para la validación de un protocolo de antibioticoterapia subcutánea paliativa • Miguel Antonio Sánchez-Cárdenas, Laura Camila Pulido-Garzón, Leydy Viviana Santamaría-Orozco, Lina Katerine Rodríguez-Laverde, Mónica Andrea Preciado-Vargas, Sergio Daniel Ruíz-Alarcón, Yeins Stiven Prado-Ariza

Bruera E, Brenneis C, Michaud M, BacovskyR, Chadwick S, Emeno A, et al.. Use of the subcutaneous route for the administration of narcotics in patients with cancer pain. Cáncer [Internet]. 1988 [consultado 20 de febrero de 2015]; 62(2): 407-11. Disponible en: http://onlinelibrary.wiley.com/doi/10.1002/1097-0142(19880715)62:2\%3C407::AIDCNCR2820620227\%3E3.0.CO;2-T/abstract

Carrasco P. Importance of evidence-based nursing in the development of protocols and clinical guidelines. MedWave [Internet]. 2004 [consultado 19 de abril de 2015]; 4(5): e2750. Disponible en: http://www.medwave.cl/link.cgi/Medwave/ Enfermeria/junio2004/2750\#

Díaz J. Guía práctica de lectura crítica de artículos científicos originales en Ciencias de la Salud. [Internet]. Ceuta, España: Instituto Nacional de Gestión Sanitaria; 2008 [consultado 15 de mayo de 2015]; 288 p. Disponible en: http:// www.ingesa.msssi.gob.es/estadEstudios/documPublica/internet/pdf/Guia_practica_de_lectura.pdf

Ferreira I, Urrútia G, Alonso P. Revisiones sistemáticas y metaanálisis: bases conceptuales e interpretación. Rev Esp Cardiol [Internet]. 2011 [consultado 25 de mayo de 2015]; 64(8): 688-696. Disponible en: http://www.revespcardiol.org/ es/revisiones-sistematicas-metaanalisis-bases-conceptuales/articulo/90024424/

Lilley L, Aucker R. Farmacología en enfermería. España: Elsevier; 1999. 873 p.

Lozano D. Evaluación crítica de la evidencia científica. Investigación y Antropología [Internet]. 1999 [consultado 20 de abril de 2015]; 1(1): 13-18. Disponible en: http://revistas.javeriana.edu.co/index.php/imagenydesarrollo/article/view/1166/660

Mulhall A. Nursing, research, and the evidence. Evid Based Nurs [Internet]. 1998 [consultado 23 de mayo de 2015]; 1(1): 4-6. Disponible en: http://ebn.bmj.com/content/1/1/4.full

Organizacion Mundial de la Salud (OMS). Cuidados Paliativos. Who.int. Internet]. 2016 [consultado 25 de febrero de 2015]. Disponible en: http://www.who.int/cancer/palliative/es/

Quispe C. ¿Es el Factor de Impacto un buen indicador para medir la calidad de las revistas científicas?: análisis de algunos problemas generados por su uso. Infobib [Internet]. 2004 [consultado 12 de mayo de 2015]; (3). Disponible en: http:// eprints.rclis.org/5002/1/articulo1.pdf

Saénz D. Principios de farmacología general y administración de fármacos. [Internet]. San José, Costa Rica: Editorial Universidad de Costa Rica; 1993 [consultado 20 de abril de 2015]; 95 p. Disponible en: https://books.google.es/books/ about/Principios de Farmacolog\%C3\%ADa_General_Y Ad.html?hl=es\&id=gU5F26XLnA4C

Shekelle PG, Woolf S, Eccles M, Grimshaw J. Developing guidelines. BMJ [Internet]. 1999 [consultado 20 de febrero de 2015]; 318(7183): 593-596. Disponible en: http://www.ncbi.nlm.nih.gov/pmc/articles/PMC1115034/

Soriano H, Rodenas L, Moreno D, Roldán B, Castaño E, Palazón E. Utilización de la Vía Subcutánea en Atención Primaria. Rev Clin Med Fam [Internet]. 2009 [consultado 12 de febrero de 2015]; 2(08): 426-433. Disponible en: http:// scielo.isciii.es/pdf/albacete/v2n8/revision2.pdf

Soto M, Rada G. Formulación de Preguntas en Medicina Basada en la Evidencia. Rev. méd. Chile [Internet]. 2003 [consultado 20 de abril de 2015]; 131(10): 1202-1203. Disponible en: http://www.scielo.cl/scielo.php?script=sci_arttext \&pid $=$ S0034-98872003001000016

Universidad de la Salle. Estrategias de búsqueda. www.evirtual.lasalle.edu.co [Internet]. 2005 [consultado 15 de mayo de 2015]. Disponible en: http://evirtual.lasalle.edu.co/info_basica/nuevos/guia/resumenNivell.pdf

Urra E, Retamal C, Tapia C, Rodríguez M. Enfermería basada en la evidencia: qué es, sus características y dilemas. Revista de investigación y educación en Enfermería [Internet]. 2010 [consultado 20 de abril de 2015]; 28(1): 108-118. Disponible en: http://www.redalyc.org/pdf/1052/105215294014.pdf

Varo J. Gestión estratégica de la calidad en los servicios sanitarios. Madrid: Diaz de Santos; 1993. 